\title{
The Enigma of the Tinnitus-Free Dream State in a Bayesian World
}

\author{
Dirk De Ridder, ${ }^{1,2}$ Kathleen Joos, ${ }^{3}$ and Sven Vanneste ${ }^{3,4}$ \\ ${ }^{1}$ Department of Surgical Sciences, Dunedin School of Medicine, University of Otago, P.O. Box 56, Dunedin 9054, New Zealand \\ ${ }^{2}$ BRAI ${ }^{2} N$ \& TRI, Sint Augustinus Hospital, Antwerp, Belgium \\ ${ }^{3}$ Department of Translational Neuroscience, Faculty of Medicine, University of Antwerp, Belgium \\ ${ }^{4}$ School of Behavioral and Brain Sciences, The University of Texas at Dallas, USA
}

Correspondence should be addressed to Dirk De Ridder; dirk.deridder@otago.ac.nz

Received 17 March 2014; Accepted 16 April 2014; Published 6 July 2014

Academic Editor: Berthold Langguth

Copyright (C) 2014 Dirk De Ridder et al. This is an open access article distributed under the Creative Commons Attribution License, which permits unrestricted use, distribution, and reproduction in any medium, provided the original work is properly cited.

There are pathophysiological, clinical, and treatment analogies between phantom limb pain and phantom sound (i.e., tinnitus). Phantom limb pain commonly is absent in dreams, and the question arises whether this is also the case for tinnitus. A questionnaire was given to 78 consecutive tinnitus patients seen at a specialized tinnitus clinic. Seventy-six patients remembered their dreams and of these 74 claim not to perceive tinnitus during their dreams (97\%). This can be most easily explained by a predictive Bayesian brain model. That is, during the awake state the brain constantly makes predictions about the environment. Tinnitus is hypothesized to be the result of a prediction error due to deafferentation, and missing input is filled in by the brain. The heuristic explanation then is that in the dream state there is no interaction with the environment and therefore no updating of the prediction error, resulting in the absence of tinnitus.

\section{Introduction}

Fundamental concepts in psychology and philosophy of the mind are the notion of sensation and perception [1]. When a stimulus produces an effect on different sensory receptors it induces sensation. Subsequent interpretation and organization of this sensory stimulus produce a meaningful experience of the world and of one's perception [1]. Although in most cases perception is conscious, perception without awareness does exist, that is, the interpretation of semantic stimuli [2]. Normally wakefulness and awareness are related; one has to be awake; that is, there has to be a certain level of consciousness to be aware of something; that is, there is content in consciousness [3]. In states of deep sleep, anesthesia, and coma there is little or no wakefulness and hence no awareness. In drowsiness and light sleep there is more awareness. However, in certain states, dissociations exist between wakefulness and awareness, such as in the vegetative state, when there is wakefulness presumably without awareness (eyes open, brain shut) [4]. In the dream state there is awareness (content in consciousness) with decreased wakefulness (level of consciousness) [3]. Dreams are succession of images, ideas, emotions, and perceptions without sensations that occur involuntarily in the mind predominantly during rapid eye movement (REM) sleep.

Nonpulsatile subjective tinnitus is considered a phantom perception [5], the conscious awareness of a percept in the absence of an external stimulus. It is characterized by the perception that the phantom sound comes from an external sound source, even though the sound might be pulled from memory $[1,6,7]$. This is reminiscent of a dream state, when there is awareness, with stimuli attributed to the external world but generated internally [8]. Whereas tinnitus can be considered a simple phantom percept, dreams could be considered complex phantom percepts, like hallucinations and hallucinosis $[9,10]$. However, in contrast to hallucinations and hallucinosis that occur during wakefulness, dreams occur during certain stages of sleep.

Stimulus-evoked auditory cortical activation does not necessarily produce conscious auditory perception [11], and 
auditory perception is possible in the absence of auditory input: more than $80 \%$ of people with normal hearing perceive phantom sounds when placed in a soundproof room [12]. Likewise, after limb amputation almost all people experience a phantom limb [13], whereas $70 \%$ suffer from severe phantom pain [13].

A clear clinical analogy exists between phantom pain and disabling tinnitus $[1,14,15]$. There are also parallels between the pathophysiology of tinnitus and pain [1], as well as in the treatment $[16,17]$. However, there are also differences between tinnitus and pain. While physiological pain is mediated via nociceptive pathways, no analogous physiological tinnitus pathways exist. This could explain why commonly available analgesics that suppress acute physiological body pain are inefficient in ameliorating tinnitus [18]. Also, medications such as antiepileptics and antidepressants, which are effective in the treatment of neuropathic pain [18], tend to be ineffective for tinnitus [19].

Many to most (33-100\%) patients who suffer from phantom limb percepts do not experience phantom limb percepts in a dream state [20-23]. This has been explained as follows $[8,21,23]$ : neural representation of the body derives from sensory and proprioceptive feedback from the body. During sleep, when the brain/mind is actively kept offline, this sensory feedback is lacking. Moreover, during REM sleep and in the absence of external inputs, dreaming could activate a set of innate or early life spatial-temporal categories [8]. So if REM sleep is a state of protoconsciousness, that is, a contextually emergent property of self-sustaining systems, the self as it appears in REM sleep dreams is no longer affected by waking experiences because it feeds from an embodied and functionally intact body scheme $[8,21]$.

In view of the pathophysiological analogy between tinnitus and pain, it can be hypothesized that tinnitus is absent in the dream state as well. We therefore explored this in a group of 78 consecutive tinnitus patients attending the Multidisciplinary Tinnitus Research Initiative Clinic at the University of Antwerp. A recently proposed pathophysiological model of phantom sound based on a predictive brain concept with Bayesian updating [24] might explain why tinnitus is not perceived during dreaming.

\section{Methods}

2.1. Participants. Seventy-eight patients (57 males and 21 females) with chronic, nonpulsatile tinnitus were included in this study with an average age of 48.78 years $(S d=12.87)$ and an average tinnitus duration of 5.74 years $(S d=6.96)$. Thirtyfive patients perceive noise-like tinnitus, while 43 patients experience pure tone tinnitus. Forty-three patients had bilateral tinnitus; 12 patients perceive tinnitus holocranially, 12 on the left side and 11 on the right side. Antwerp University Ethics Committee reviewed and approved the study. All patients signed an approved informed consent in order to enroll into the study.

2.2. Questionnaire. A questionnaire was created based on previous research in phantom limb pain and dreaming [20].
The first question asked whether the tinnitus patient recalled if they dreamed during the night (1), followed by the question whether in their dreams they perceive tinnitus (2).

\section{Results}

Of the 78 participating patients only $2(2.56 \%)$ declared that they do not recall their dreams, while $76(97.44 \%)$ do. Of those 76 patients that do recall their dreams $74(97.73 \%)$ state that they do not perceive tinnitus while dreaming or are not aware of having tinnitus during sleep.

\section{Discussion}

People with tinnitus do not perceive tinnitus in their dreams analogous to what is reported for many phantom limb perceptions $[21,25]$. Dreams and wakefulness are both associated with awareness, but in one state of awareness there is no tinnitus (dreams), whereas in the other (wakefulness) there is tinnitus.

The reason why patients with tinnitus do not perceive tinnitus in their dream state can be theoretically explained by the Bayesian brain model which has been used as an explanation for the development of tinnitus in relation to auditory deafferentation [24]. This Bayesian brain model is founded on an extension of a predictive brain model (see Figure 1(a)).

Whereas other models (see [26] for an overview) can explain the tinnitus in the presence of deafferentation, they cannot explain why it would be absent in the dream state. The Bayesian model is compatible with both the deafferentation and noise-cancelling models [24] and provides a rationale why tinnitus develops in a wake state and not in a dream state. Previously proposed models rather describe how tinnitus would develop.

Physiologically the brain can be conceptualized as a Helmholtz machine [27] that constantly makes one or possibly multiple [28] predictions about the world. A Helmholtz machine tries to find a hidden structure in unlabeled data. Since the examples given to the learner are unlabeled, there is no error or reward signal to evaluate a potential solution; in other words, there is no updating of the predictions. A Bayesian brain however updates predictions based on what it actively explores in the environment by means of the senses $[24,29,30]$. Bayesian inference can therefore be conceptualized in a way that would be familiar to John Hughlings-Jackson as using sensory information from the environment to update memory-based expectations (held before acquiring sensory inputs) to produce posterior beliefs represented as percepts. This mechanism permits decision making based on predictions updated by actively sampling the environment for confirmation or rejection of expectations (see Figure 1(b)) [24].

Auditory deafferentation limits the amount of information the brain can acquire to make sense of the world. The topographically specific deafferentation induces a topographically specific prediction error hypothetically based on temporal incongruity [1]. In other words, it is inconsistent 


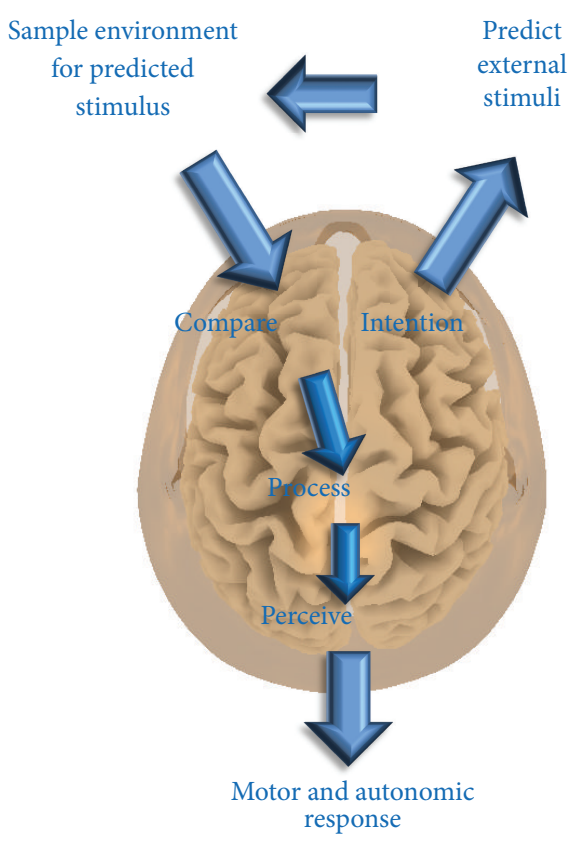

(a)

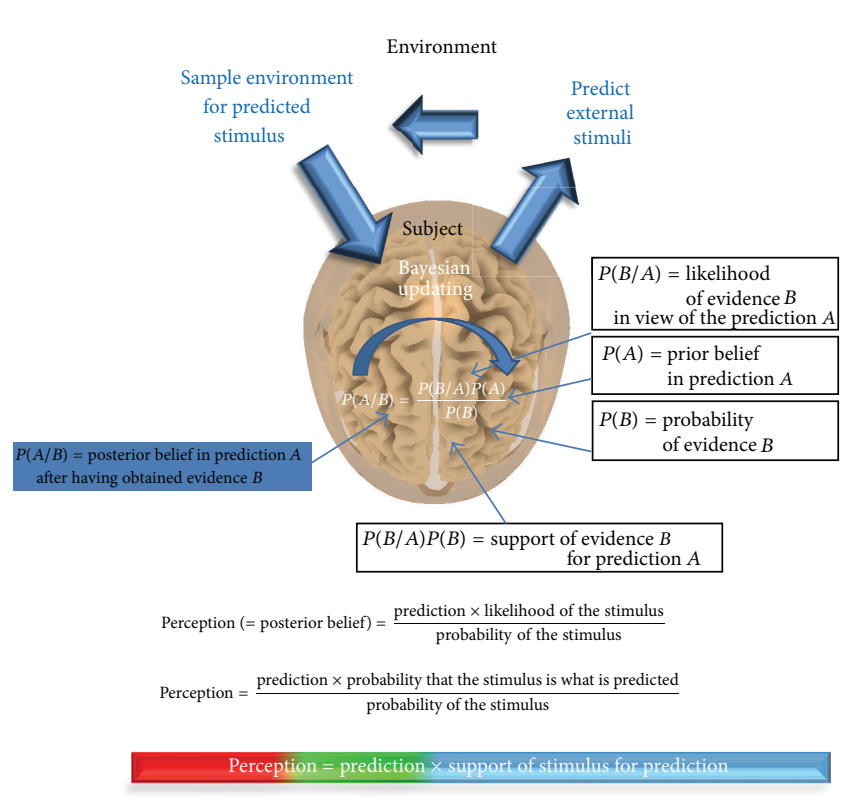

(b)

FiguRE 1: (a) The concept of the predictive brain; (b) the concept of Bayesian updating.

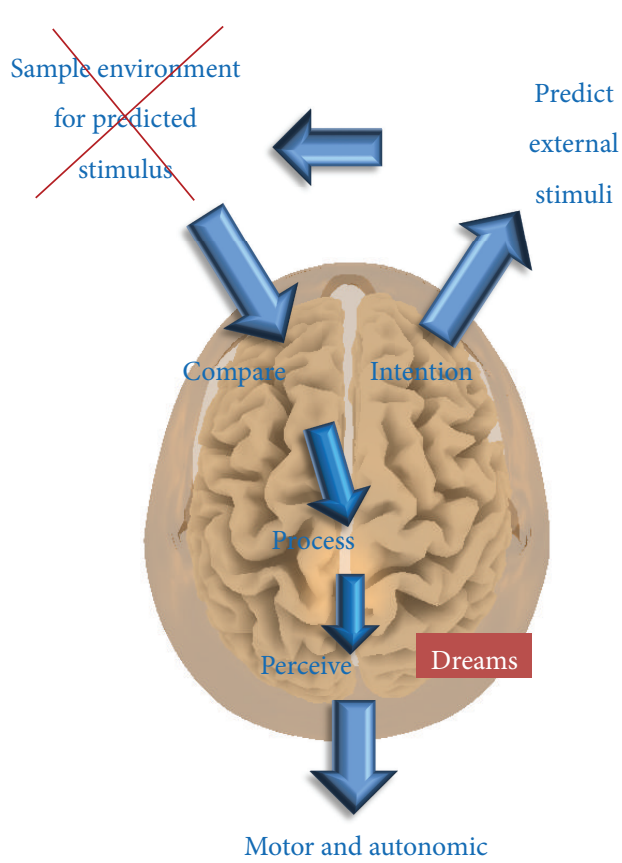

response

(a)



(b)

Figure 2: (a) Hypothetical explanation of the absence of tinnitus in dreams as seen from the predictive brain; (b) hypothetical explanation of the absence of tinnitus in dreams as seen from the Bayesian brain. 
with what is stored in memory and should be updated. The model hypothesizes that deprived auditory information depends on the amount (bandwidth) of deafferented auditory channels [24]. Limited damage to auditory receptors causes loss of functional surround inhibition in the cortex, unmasking of latent inputs, and significantly altered neural coding. However, these changes do not lead to plasticity of the cortical map [31]. This suggests that the missing information can be obtained via access of overlapping tuning curves of the neighboring cortical cells. If the deafferentation is somewhat larger, a widening of auditory receptive fields [32] will permit pulling the missing information from the auditory cortical neighborhood. If this is insufficient, due to a still larger deafferentation, dendritic and axonal rewiring can occur [33]. If this is still insufficient, the missing auditory information can be pulled from (para)hippocampal memory [24].

When we dream, we create an image of the world that is entirely detached from sensory feedback [34]; that is, it cannot be updated. This is under influence of decrease in monoamines in REM sleep. Aminergic activity is highest during waking, declines during NREM sleep, and is lowest during REM sleep. Cholinergic activity on the other hand shows the reverse pattern [34]. Sensory prediction errors are suppressed by aminergic influence during sleep [34]. This means that the discrepancy between top-down predictions and (the absence of) sensory signals received will not be registered, and the auditory deafferentation will not be filled in, resulting in the absence of tinnitus in the dream state (see Figures 2(a) and 2(b)) [26].

Indirect arguments for this hypothesis come from recent research on cerebellar influences in tinnitus. It has been argued that the cerebellum is involved in motor, sensory, and cognitive predictions [35]. It is therefore possible that auditory predictions are made in the paraflocculus, as removing this cerebellar structure can prevent tinnitus from arising and arrest the presence of tinnitus in animals [36]. This conceptually suggests that removing the prediction can prevent or abolish tinnitus, which is in accordance with the concept that tinnitus could be a malprediction [1].

However, apart from its theoretical implications, the data might also help to find the neural correlates of tinnitus. The putative on/off switch for tinnitus is to be found in these areas that differ between waking and REM state [26], that is, the ventrolateral prefrontal cortex/frontopolarinferior parietal-cerebellar-parahippocampal network [10]. These areas overlap with a recent meta-analysis of PET studies in tinnitus [37] and provide a framework for zooming in on the pathophysiology of this enigmatic symptom.

In addition to its evident benefit for tinnitus research, it could also provide clues for consciousness research, by delineating the core areas involved in the neural correlates of consciousness; that is, minimal assembly of brain areas required for consciousness per se $[38,39]$.

Other potential explanations for the absence of tinnitus in the dream state have to be considered. It is possible that during the dream state there is an attention shift from the tinnitus to the dream, analogous to what is noted in patients who do not perceive their tinnitus when intensely engaged in a task.
In conclusion, this report demonstrates that tinnitus perception is switched off during dream sleep even though there is awareness, like in wakefulness. This suggests that it is theoretically possible to find the neural correlates of phantom sound and thereby find a potential avenue for suppressing this enigmatic symptom.

\section{Conflict of Interests}

The authors declare that there is no conflict of interests regarding the publication of this paper.

\section{References}

[1] D. de Ridder, A. B. Elgoyhen, R. Romo, and B. Langguth, "Phantom percepts: tinnitus and pain as persisting aversive memory networks," Proceedings of the National Academy of Sciences of the United States of America, vol. 108, no. 20, pp. 8075-8080, 2011.

[2] S. Dehaene, L. Naccache, G. Le Clec'H et al., "Imaging unconscious semantic priming," Nature, vol. 395, no. 6702, pp. 597600, 1998.

[3] S. Laureys, “The neural correlate of (un)awareness: Lessons from the vegetative state," Trends in Cognitive Sciences, vol. 9, no. 12, pp. 556-559, 2005.

[4] S. Laureys, "Eyes open, brain shut," Scientific American, vol. 296, no. 5, pp. 84-89, 2007.

[5] P. J. Jastreboff, "Phantom auditory perception (tinnitus): mechanisms of generation and perception," Neuroscience Research, vol. 8, no. 4, pp. 221-254, 1990.

[6] A. Shulman, "A final common pathway for tinnitus-the medial temporal lobe system," The International Tinnitus Journal, vol. 1, no. 2, pp. 115-126, 1995.

[7] D. de Ridder, H. Fransen, O. Francois, S. Sunaert, S. Kovacs, and P. Van De Heyning, "Amygdalohippocampal involvement in tinnitus and auditory memory," Acta oto-laryngologica. Supplementum, no. 556, pp. 50-53, 2006.

[8] J. A. Hobson, "REM sleep and dreaming: towards a theory of protoconsciousness," Nature Reviews Neuroscience, vol. 10, no. 11, pp. 803-814, 2009.

[9] S. Vanneste, J. J. Song, and D. de Ridder, "Tinnitus and musical hallucinosis: the same but more," Neuroimage, vol. 82, pp. 373383, 2013.

[10] J. A. Hobson and E. F. Pace-Schott, "The cognitive neuroscience of sleep: neuronal systems, consciousness and learning," Nature Reviews Neuroscience, vol. 3, no. 9, pp. 679-693, 2002.

[11] B. W. Colder and L. Tanenbaum, "Dissociation of fMRI activation and awareness in auditory perception task," Cognitive Brain Research, vol. 8, no. 3, pp. 177-184, 1999.

[12] L. del Bo, S. Forti, U. Ambrosetti et al., "Tinnitus aurium in persons with normal hearing: 55 years later," OtolaryngologyHead and Neck Surgery, vol. 139, no. 3, pp. 391-394, 2008.

[13] H. Flor, L. Nikolajsen, and T. S. Jensen, "Phantom limb pain: a case of maladaptive CNS plasticity?" Nature Reviews Neuroscience, vol. 7, no. 11, pp. 873-881, 2006.

[14] J. Tonndorf, "The analogy between tinnitus and pain: a suggestion for a physiological basis of chronic tinnitus," Hearing Research, vol. 28, no. 2-3, pp. 271-275, 1987.

[15] A. R. Moøller, "Similarities between chronic pain and tinnitus," The American Journal of Otology, vol. 18, no. 5, pp. 577-585, 1997. 
[16] D. de Ridder, G. de Mulder, T. Menovsky, S. Sunaert, and S. Kovacs, "Electrical stimulation of auditory and somatosensory cortices for treatment of tinnitus and pain," Progress in Brain Research, vol. 166, pp. 377-388, 2007.

[17] D. De Ridder, S. Vanneste, T. Menovsky, and B. Langguth, "Surgical brain modulation for tinnitus: the past, present and future," Journal of Neurosurgical Sciences, vol. 56, no. 4, pp. 323340, 2012.

[18] D. De Ridder and A. Moller, "Similarities between treatments of tinnitus and central pain," in Textbook of Tinnitus, A. Moller, B. Langguth, D. De Ridder, and T. Kleinjung, Eds., pp. 753-762, Springer, New York, NY, USA, 2011.

[19] A. B. Elgoyhen and B. Langguth, "Pharmacological approaches to the treatment of tinnitus," Drug Discovery Today, vol. 15, no. 7-8, pp. 300-305, 2009.

[20] P. Brugger, "The phantom limb in dreams," Consciousness and Cognition, vol. 17, no. 4, pp. 1272-1280, 2008.

[21] M. Alessandria, R. Vetrugno, P. Cortelli, and P. Montagna, "Normal body scheme and absent phantom limb experience in amputees while dreaming," Consciousness and Cognition, vol. 20, no. 4, pp. 1831-1834, 2011.

[22] B. Frank and E. Lorenzoni, "Experiences of phantom limb sensations in dreams," Psychopathology, vol. 22, no. 4, pp. 182187, 1989.

[23] R. Vetrugno, I. Arnulf, and P. Montagna, "Disappearance of "phantom limb" and amputated arm usage during dreaming in REM sleep behaviour disorder," BMJ Case Reports, 2009.

[24] D. De Ridder, S. Vanneste, and W. Freeman, "The Bayesian brain: phantom percepts resolve sensory uncertainty," Neuroscience \& Biobehavioral Reviews, 2012.

[25] T. Mulder, J. Hochstenbach, P. U. Dijkstra, and J. H. B. Geertzen, "Born to adapt, but not in your dreams," Consciousness and Cognition, vol. 17, no. 4, pp. 1266-1271, 2008.

[26] D. de Ridder, S. Vanneste, N. Weisz et al., "An integrative model of auditory phantom perception: Tinnitus as a unified percept of interacting separable subnetworks," Neuroscience and Biobehavioral Reviews, 2013.

[27] P. Dayan, G. E. Hinton, R. M. Neal, and R. S. Zemel, "The Helmholtz machine," Neural Computation, vol. 7, no. 5, pp. 889904, 1995.

[28] D. de Ridder, J. Verplaetse, and S. Vanneste, "The predictive brain and the "free will" illusion," Frontiers in Psychology, vol. 4, p. 131, 2013.

[29] K. Friston, “The free-energy principle: a unified brain theory?” Nature Reviews Neuroscience, vol. 11, no. 2, pp. 127-138, 2010.

[30] D. C. Knill and A. Pouget, "The Bayesian brain: the role of uncertainty in neural coding and computation," Trends in Neurosciences, vol. 27, no. 12, pp. 712-719, 2004.

[31] R. Rajan, "Receptor organ damage causes loss of cortical surround inhibition without topographic map plasticity," Nature Neuroscience, vol. 1, no. 2, pp. 138-143, 1998.

[32] L. Chen, P. G. Trautwein, M. Shero, and R. J. Salvi, “Tuning, spontaneous activity and tonotopic map in chicken cochlear ganglion neurons following sound-induced hair cell loss and regeneration," Hearing Research, vol. 98, no. 1-2, pp. 152-164, 1996.

[33] C. Y. Hsieh, C. T. Hong, and K. S. Cramer, "Deletion of EphA4 enhances deafferentation-induced ipsilateral sprouting in auditory brainstem projections," Journal of Comparative Neurology, vol. 504, no. 5, pp. 508-518, 2007.
[34] J. A. Hobson and K. J. Friston, "Waking and dreaming consciousness: neurobiological and functional considerations," Progress in Neurobiology, vol. 98, no. 1, pp. 82-98, 2012.

[35] N. Ramnani, "The primate cortico-cerebellar system: anatomy and function," Nature Reviews Neuroscience, vol. 7, no. 7, pp. 511$522,2006$.

[36] C. A. Bauer, W. Kurt, L. T. Sybert, and T. J. Brozoski, "The cerebellum as a novel tinnitus generator," Hearing Research, vol. 295, pp. 130-139, 2013.

[37] J. J. Song, D. de Ridder, P. van de Heyning, and S. Vanneste, "Mapping tinnitus-related brain activation: an activationlikelihood estimation metaanalysis of PET studies," Journal of Nuclear Medicine, vol. 53, no. 10, pp. 1550-1557, 2012.

[38] F. Crick and C. Koch, "A framework for consciousness," Nature Neuroscience, vol. 6, no. 2, pp. 119-126, 2003.

[39] F. Crick and C. Koch, "Are we aware of neural activity in primary visual cortex?” Nature, vol. 375, no. 6527, pp. 121-123, 1995. 

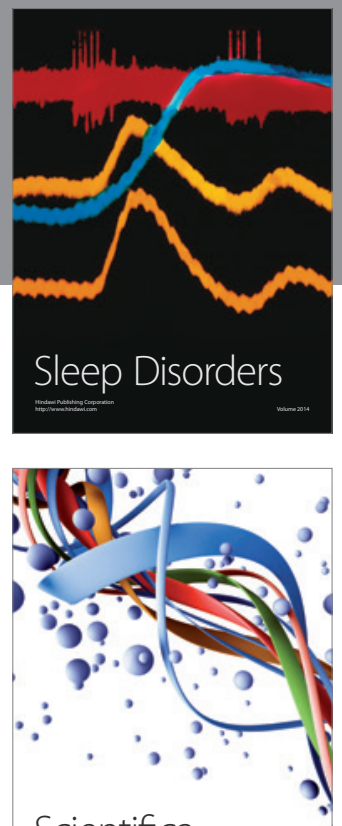

Scientifica
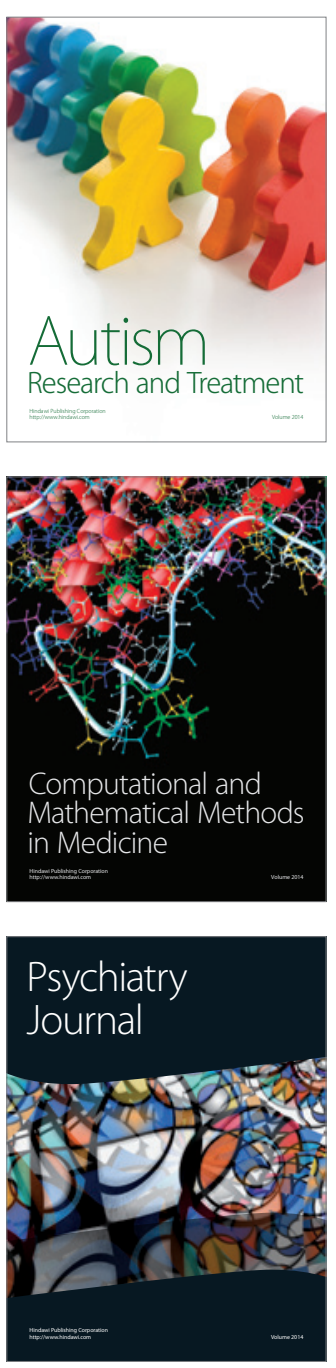
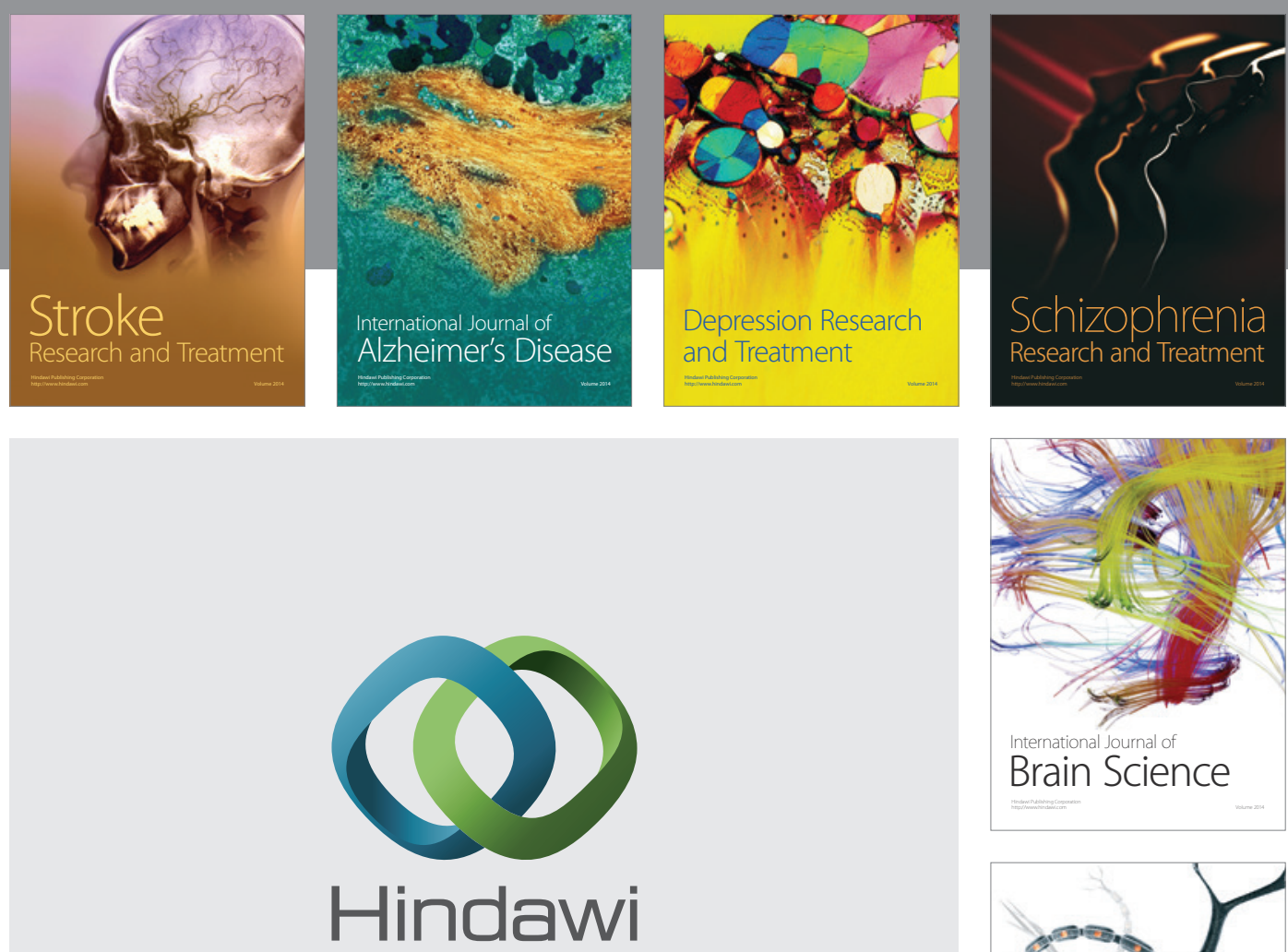

Submit your manuscripts at

http://www.hindawi.com
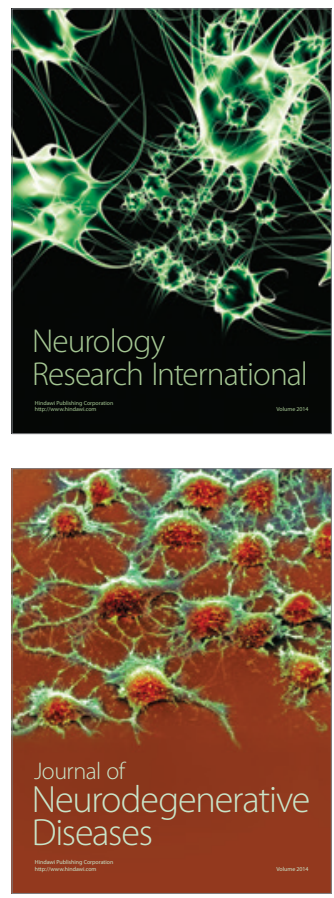

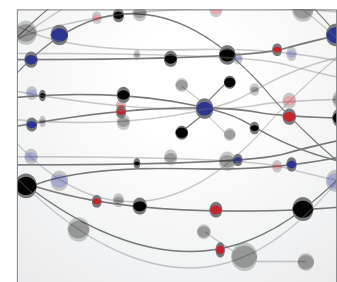

The Scientific World Journal
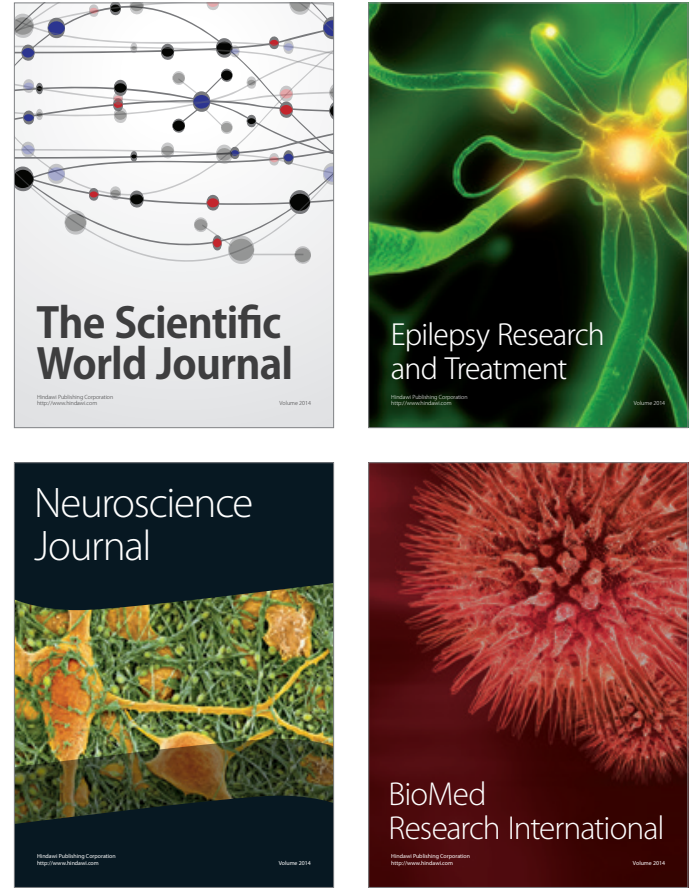



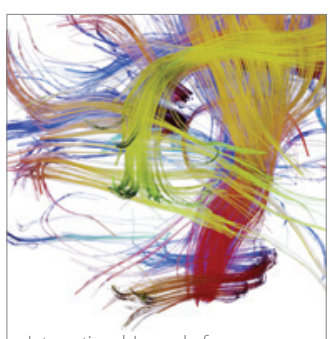

Brain Science

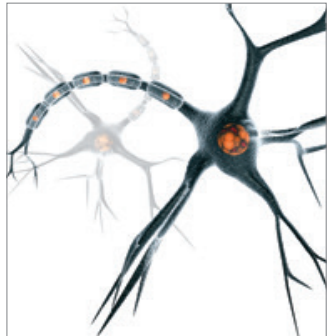

Neural Plasticity
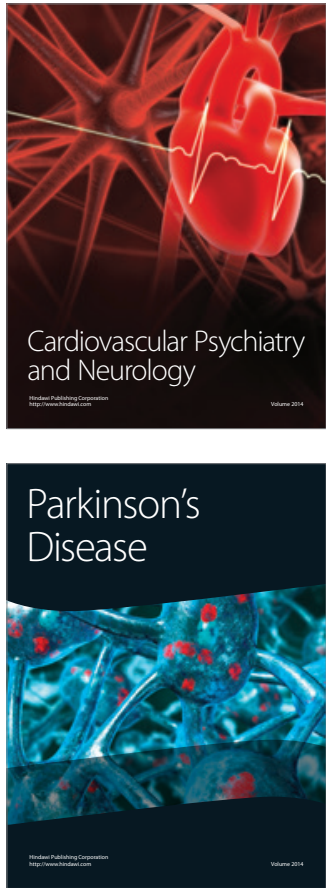